\title{
Stagnation-Point Flow of Magneto-Williamson Nanofluid over a Stretching Material with Ohmic Heating and Entropy Analysis
}

\author{
Ephesus Olusoji Fatunmbi ${ }^{1}$, Fazle Mabood ${ }^{2}$, Adetunji Adeniyan ${ }^{3}$
}

1. Department of Mathematics and Statistics, Federal Polytechnic, Ilaro, Nigeria.

2. Department of Information Technology, Fanshawe College London, ON Canada.

3. Department of Mathematics, University of Lagos, Nigeria.

*Corresponding author: ephesus.fatunmbi@federalpolyilaro.edu.ng

\section{Article Info}

Received: 28 July 2021

Accepted: 14 October 2021
Revised: 13 October 2021

Available online: 20 October 2021

\begin{abstract}
This study communicates stagnation-point flow in magneto-Williamson nanofluid along a convectively heated nonlinear stretchable material in a porous medium. The impacts of Joule heating, thermophoresis together with Brownian motion are also checked in this investigation. In addition, thermodynamic second law is applied to develop entropy generation analysis of crucial parameters with identification of parameters capable of minimizing energy loss in the system. The transport equations are simplified into ordinary differential equations and then integrated numerically using Runge-Kutta-Fehlberg with shooting technique. The effects of the emerging parameters on the dimensionless velocity, temperature, concentration and entropy generation number are publicized through tables and graphs with appropriate discussions. In the limiting conditions, the results are found to conform accurately with published studies in the literature. It is found that the viscous drag can be reduced by lowering the magnitude of Weissenberg number, magnetic field and Darcy parameters while heat transfer at the surface improves in the presence of surface convection, temperature ratio and thermal radiation parameters. Besides, the analysis reveals that entropy generation can be minimized by lowering the magnitude of magnetic field, Schmidt number and surface convection parameters. The reduction in these parameters will promote efficient performance of thermal devices. More so, the results obtained in this study can be useful for the construction of appropriate thermal devices for use in energy and electronic devices.
\end{abstract}

Keywords: Porous medium; Entropy generation; Thermophoresis; Stagnation-point flow; Williamson fluid.

MSC2010:35Q20

\section{Introduction}

Reports on the flow and heat transfer of non-Newtonian fluids have become more fascinating due to widespread applications in various fields of science, engineering and technology. Such fluids 
are frequently found in crude oil drilling, slurry technology, paints production, drug and pharmaceuticals, food processing and manufacturing, cosmetics and lubricants, molten plastic material manufacturing, etc. [1]. These fluids differ sharply from the Newton's law of viscosity and theory of Navier-Stokes model. Thus, various models of non-Newtonian fluids are available in literature as their properties cannot be captured by a single constitutive model. Some of these models are: Micropolar fluid, Casson fluid, Eyring-Powell, Giesekus, tangent hyperbolic fluid, Maxwell, power-law and Williamson fluid models. The latter has a unique characteristics of shear thinning attributes in which higher rates of shear stress reduces fluid viscosity, Williamson [2]. The motion of plasma, emulsion sheet and blood flows can be described by this model. The boundary layer flow of such fluid was initiated by Nadeem et al. [3] over a stretching sheet. Shah et al. [4] scrutinized an unsteady transport of Williamson fluid over a porous stretching sheet coupled with the influence of thermal radiation whereas the flow of Williamson fluid between two rotating disks was carried out by Qayyum et al. [5] with entropy generation analysis while Aldabesh et al. [6] recently discussed such a concept on a rotating cylinder with the inclusion of suspended nanoparticles, thermal radiation, variable thermal conductivity and gyrotactic microorganisms.

The investigation of boundary layer flow induced by stretched surfaces has been widely publicized by researchers and scientists due to various applications derivable from such studies. Various applications can be found in polymer manufacturing industries such as wire drawing, metal as well as extrusion of polymer, hot rolling, textile and paper production, cooling of the metallic sheet and so on. The initial investigation of such a concept was done by Crane [7]. The author reported a closed-form exact solution for time-independent boundary layer flow prompted by a flat stretching material. In view of the applications of such investigations, various authors have reported the extended and improved version in the literature considering different geometries, conditions and parameters (see [8-11]). However, the case of nonlinear stretching sheet which has practical usefulness engineering and material manufacturing processes has not receive proper attention in the literature. Therefore, it becomes necessary to investigate such a phenomenon for practical purposes as studied by $[12-15]$ on different flow configurations and conditions.

Nanofluid represents a class of fluid with an improved thermal conductivity in comparison to the conventional base fluids (water, oil, ethylene glycol, etc). It is made of minutes nanoparticles consisting of metals, oxides, etc. The applications of nanofluid abound in engineering and industrial works for enhancement of cooling process in high-energy equipment such as in power manufacturing and atomic reactors, transportation processes, pharmaceutical industries, industrial cooling, etc. In the light of these applications, Noor et al. [17] scrutinized the motion of a micropolar nanofluid towards a stagnation point configured in vertically stretched surface. Aly and Pop [18] theoretically examined time-independent flow and heat dissipation of magnetohydrodynamic nanofluid towards a stagnation point with slip condition. Likewise, Anuar et al. [19] numerically discussed such a problem over a convectively heated stretching/shrinking material while Fatunmbi and Okoya [20] recently deliberated on the flow of nonlinear mixed convection Casson fluid with nanoparticles suspension in the neighbourhood of a stagnation point.

The impact of thermal radiation in engineering operations is crucial especially in solar power technology, gas turbines, hot rolling and so on. In the presence high-temperature difference in the flow field, it is important to apply the nonlinear thermal radiation for better prediction [21-22]. Flow in porous medium associated with magnetic field gains much relevance in diverse engineering processes such as MHD generators, crude oil extraction, irrigation systems, etc. In view of these applications, Mukhopadhyay et al. [23] presented a numerical report on the transport of a Newtonian fluid enclosed in a porous medium with nonlinear radiation effect. This concept has also been studied by various authors investigating different configurations and conditions as found in Refs [24-26].

Entropy production can be described as the quantity of entropy that is generated in any irreversible process. It is the measure of randomness and the magnitudes of the irreversibility occurring in a system. The subject of entropy generation corresponds to thermodynamic irreversibility was initially investigated by Bejan [27-28] who utilized the basic principles of thermodynamics to study heat transfer and thermal design processes. Due to these innumerable applications, Salawu and Ogunseye [29] studied the problem in the presence of chemical reaction using Eyring-Powell fluid 
while MHD second grade nanofluid was employed by Sithole et al. [30] to examine such a concept whereas Mondal et al. [31] addressed such phenomenon with the transport of a dusty fluid alongside with variable viscosity and radiation effects. More so, the unsteady case of such problems with Casson fluid was conducted by Shit and Mandal [32], Fatunmbi and Salawu [33] studied such effects with hydromagnetic micropolar fluid with non-homogenous heat generation while Alsaadi et al. [34] reported such phenomenon engaging electrically Williamson nanofluid being influenced by activation energy. Recently, a numerical investigation of entropy generation analysis in a dissipative magnetohydrodynamic micropolar fluid flow induced by a nonlinear stretchable surface was carried out by Fatunmbi and Salawu [35] with the impact variable magnetic field and dynamic viscosity. The authors stated that growth in the thermal radiation parameter caused the heat transfer irreversibility to dominate viscous dissipation irreversibility. Also, Fatunmbi and Adeniyan [36] reported entropy generation on steady magneto-micropolar fluid flow over a nonlinear stretching sheet with nonlinear thermal radiation. The analysis showed that Eckert and Prandtl numbers enhanced generation of entropy in the system.

Motivated by the aforementioned studies coupled with various applications highlighted above, the aim of the present study is to investigate the motion and heat transfer of magnetohydrodynamic Williamson nanofluid induced by a convectively heated nonlinear stretching sheet in a porous medium with entropy analysis. The flow is in the neighbourhood of a stagnation point and characterized by temperature-dependent material properties (viscosity and thermal conductivity) with the inclusion of crucial engineering parameters such as nonlinear thermal radiation, joule heating, viscous dissipation, thermophoresis and Brownian motion. The main equations are solved numerically via shooting technique coupled with Runge-Kutta Fehlberg scheme.

\section{Problem formulation}

Consider an incompressible and steady motion of magnetohydrodynamic Williamson nanofluid in a two-dimensional stretching sheet configured in a porous medium. The flow configuration is characterized by $(x, y)$ coordinate with respective velocity component $(u, v)$ in which $x$ axis depicts the flow direction with $y$ axis perpendicular to it. The sheet is stretched by the application of an equal but opposing force, the origin is maintained at point $y=0$ as sketched in Fig. 1. At the sheet, the velocity is $u=U_{w}=b x^{r}$ whereas the upstream velocity is $u \rightarrow U_{e}=c x^{r} \quad(b>0$ indicates stretching rate and $c$ is a constant which measures the magnitude of stagnation point flow while $r$ is the power law index which relates to the surface stretching speed). An external magnetic field with magnitude $B(x)=B_{0} x^{(r-1) / 2}[36,37]$ is applied normal to the axis of flow, the induced magnetic field is ignored. The sheet is convectively heated at the bottom from a hot fluid having a temperature $T_{f}$ and the heat transfer coefficient is expressed as $h_{T}=h_{f} x^{(r-1) / 2}$ whereas the non-uniform permeability of the porous medium is specified as $K_{p}=K_{0} x^{1-r}[24,38]$. Fluid viscosity and thermal conductivity are assumed to be temperature-dependent while other fluid characteristics are taken to be constant. The impacts of thermophoresis, Brownian motion and nonlinear thermal radiation are also checked in the heat transfer field. 


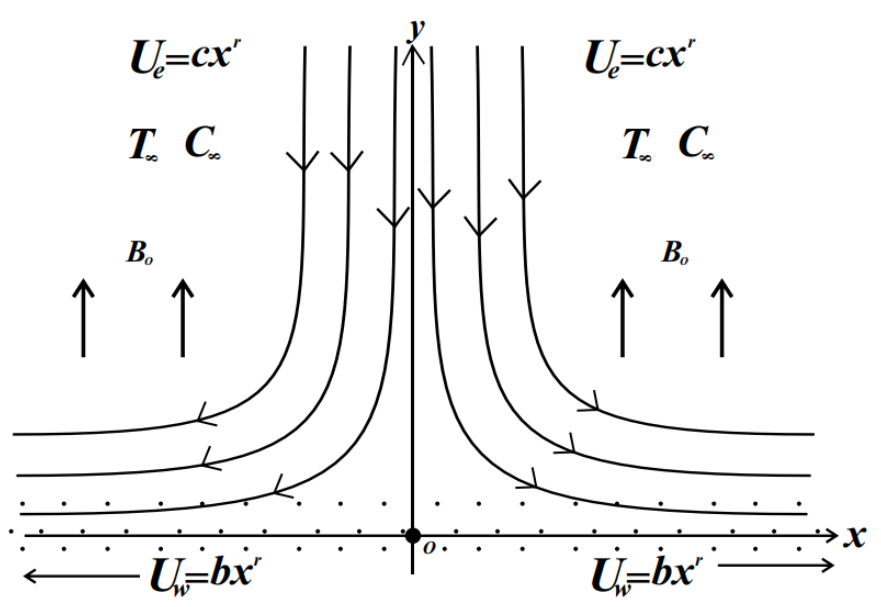

Fig. 1 The Flow Geometry

Incorporating the aforementioned assumptions and the famous boundary layer approximation, the equations governing the motion and heat transfer of Williamson fluid are specified below (see [34-39]).

$$
\begin{gathered}
\frac{\partial u}{\partial x}+\frac{\partial v}{\partial y}=0 \\
\rho_{f_{\infty}}\left(u \frac{\partial u}{\partial x}+v \frac{\partial u}{\partial y}\right)=U_{e} \frac{d U_{e}}{d x}+\frac{\partial}{\partial y}\left(\mu(T) \frac{\partial u}{\partial y}+\sqrt{2} \Gamma \mu(T)\left(\frac{\partial u}{\partial y}\right)^{2}\right)-\frac{\mu(T)}{K_{p}}\left(u-U_{e}\right)-\sigma B(x)^{2}\left(u-U_{e}\right) \\
u \frac{\partial T}{\partial x}+v \frac{\partial T}{\partial y}=\frac{1}{\left(\rho_{\infty} c_{p}\right)_{f}} \frac{\partial}{\partial y}\left(k \frac{\partial T}{\partial y}\right)+\frac{\left(\rho_{\infty} c\right)_{p}}{\left(\rho_{\infty} c\right)_{f}}\left[\frac{D_{T}}{T_{\infty}}\left(\frac{\partial T}{\partial y}\right)^{2}+D_{B}\left(\frac{\partial T}{\partial y} \frac{\partial C}{\partial y}\right)\right]+ \\
\frac{1}{\left(\rho_{\infty} c_{p}\right)_{f}}\left(\sigma B(x)^{2}+\frac{\mu(T)}{K_{p}}\right)\left(u-U_{e}\right)^{2}+\frac{\mu(T)}{\left(\rho_{\infty} c_{p}\right)_{f}}\left(\left(\frac{\partial u}{\partial y}\right)^{2}+\Gamma\left(\frac{\partial u}{\partial y}\right)^{3}\right)+ \\
\frac{16 \sigma^{\star}}{3 k_{\star}\left(\rho_{\infty} c_{p}\right)_{f}} \frac{\partial}{\partial y}\left(T^{3} \frac{\partial T}{\partial y}\right) \\
u \frac{\partial C}{\partial x}+v \frac{\partial C}{\partial y}=D_{B} \frac{\partial C^{2}}{\partial y^{2}}+\frac{D_{T}}{T_{\infty}}\left(\frac{\partial^{2} T}{\partial y^{2}}\right)
\end{gathered}
$$

The conditions at the wall are specified as (see Isede and Adeniyan [40]):

$$
\begin{aligned}
& v=v(x), \text { along the } y-\text { axis } \\
& u=U_{w}=b x^{r}, v=0,-k_{\infty} \frac{\partial T}{\partial y}=h_{T}\left(T_{f}-T_{\infty}\right), C=C_{w} \text { at } y=0, \\
& u \rightarrow U_{e}=c x^{r}, T \rightarrow T_{\infty}, C \rightarrow \infty \text { as } y \rightarrow \infty .
\end{aligned}
$$

The temperature-dependent viscosity and thermal conductivity are sequentially written as [41]

$$
\mu=\mu_{\infty} e^{-\alpha \theta}, k=k_{\infty}(1+\varepsilon \theta),
$$

with $\mu_{\infty}, k_{\infty}, \varepsilon$ and $\alpha$ orderly indicates the free stream viscosity, upstream thermal conductivity, thermal conductivity parameter and viscosity parameter. 


\subsection{The Entropy Generation Equation}

In view of thermodynamics second law, the volumetric entropy generation for the magneto-Williamson nanofluid in the presence of viscous dissipation, magnetic field effect, nonlinear thermal radiation and diffusion influence is expressed in Eq. (2.7) (see [36,42-44]).

$$
S_{\text {gen }}=S_{h}+S_{v}+S_{m}+S_{d}
$$

where $S_{g e n}$ is the volumetric entropy generation, $S_{h}$ is the entropy production resulting from heat transfer or the conduction effect, $S_{v}$ denotes entropy resulting from viscous dissipation, $S_{m}$ entropy generation owing to magnetic field or Joule heating effect and $S_{d}$ represents entropy production due to diffusion effect. The respective terms in Eq. (2.7) are presented in (2.8) as follows:

$$
\begin{aligned}
& S_{\text {gen }}=\frac{1}{T^{2}}\left(k+\frac{16 \sigma^{\star} T^{3}}{3 k^{\star}}\right)\left(\frac{\partial T}{\partial y}\right)^{2}+\frac{\mu}{T}\left(\left(\frac{\partial u}{\partial y}\right)^{2}+\Gamma\left(\frac{\partial u}{\partial y}\right)^{3}\right)+ \\
& \frac{1}{T}\left(\sigma B(x)^{2}+\frac{\mu(T)}{K_{p}}\right) u^{2}+D_{B}\left(\frac{\partial C}{\partial y}\right)\left(\frac{1}{C} \frac{\partial C}{\partial y}+\frac{1}{T} \frac{\partial T}{\partial y}\right) .
\end{aligned}
$$

In the governing equations described above, $u$ and $v$ represent velocity components in $x$ and $y$ directions, while $\rho_{\infty}, \mu_{\infty}, \sigma,\left(\rho c_{p}\right)_{f}$ and $\left(\rho c_{p}\right)_{p}$ respectively indicates ambient fluid density, ambient dynamic viscosity, electrical conductivity, heat capacity of fluid and heat capacity of nanoparticle respectively. Likewise, $\Gamma, D_{B}, T, C, T_{\infty}, C_{\infty}, D_{T}, U_{w}, U_{e}, \sigma^{\star}, k^{\star}, k$ and $S_{g e n}$ respectively indicates relaxation time, Brownian diffusion coefficient, fluid temperature, fluid concentration, ambient temperature, ambient concentration, thermophoretic diffusion coefficient, velocity at the wall, upstream velocity, Stefan-Boltzmann constant, mean absorption coefficient, thermal conductivity and volumetric entropy generation. With the introduction of the quantities in Eq. (2.9), the transport equations have been transformed into ordinary differential Eqs. [39].

$$
\eta=\sqrt{\frac{b x^{r-1}}{\nu_{\infty}}} y, \psi=\sqrt{\nu_{\infty} b x^{r+1}} f(\eta), \theta(\eta)=\frac{T-T_{\infty}}{T_{f}-T_{\infty}}, \phi(\eta)=\frac{C-C_{\infty}}{C_{f}-C_{\infty}} .
$$

Hence, in view of Eq. (2.9) in (2.1-2.5) and taking cognizance of Eq. (2.6), then Eq. automatically becomes valid while Eqs. (2.2-2.4) result to:

$$
\begin{gathered}
e^{-\alpha \theta}\left[\left(1+W e f^{\prime \prime}\right) f^{\prime \prime \prime}-\alpha f^{\prime \prime} \theta^{\prime}\left(1+W e f^{\prime \prime}\right)\right]+\frac{r+1}{2} f f^{\prime \prime}-r f^{\prime 2}+r H^{2}-\left(M+D_{a} e^{-\alpha \theta}\right)\left(f^{\prime}-H\right)=0, \\
{\left[1+\varepsilon \theta+N r\left(1+\left(\theta_{w}-1\right) \theta\right)^{3}\right] \theta^{\prime \prime}+3 N r\left(\theta_{w}-1\right) \theta^{\prime 2}\left(1+\left(\theta_{w}-1\right) \theta\right)^{2}+\varepsilon \theta^{\prime 2}+\frac{r+1}{2} \operatorname{Pr} f \theta^{\prime}+} \\
e^{-\alpha \theta} \operatorname{Pr} E c\left(1+\frac{W e}{\sqrt{2}} f^{\prime \prime}\right) f^{\prime \prime 2}+\operatorname{Pr}\left(N_{t} \theta^{\prime 2}+N_{b} \theta^{\prime} \phi^{\prime}\right)+\operatorname{PrEc}\left(M+D_{a} e^{-\alpha \theta}\right)\left(f^{\prime}-H\right)^{2}=0, \\
\phi^{\prime \prime}+\frac{N_{t}}{N_{b}} \theta^{\prime \prime}+\frac{r+1}{2} S c f \phi^{\prime}=0,
\end{gathered}
$$

The wall conditions also result to:

$$
\begin{aligned}
& f^{\prime}(0)=1, f(0)=0,0^{\prime}(0)=-\zeta(1-\theta(0)), \phi(0)=1 \\
& f^{\prime}(\infty)=H, \theta(\infty)=0, \phi(\infty)=0 .
\end{aligned}
$$

Furthermore, the entropy generation Eq.(2.7) in its simplified form (with $r=1$ ) yields

$$
\begin{aligned}
& N_{G}=\frac{\operatorname{Re}\left(\theta_{w}-1\right)^{2}\left[1+\epsilon \theta+N r\left(1+\left(\theta_{w}-1\right) \theta\right)^{3}\right]}{\left(1+\left(\theta_{w}-1\right) \theta\right)^{2}} \theta^{\prime 2}+\frac{\left(f^{\prime \prime}+\frac{W e}{\sqrt{2}} f^{\prime \prime 3}\right) e^{-\alpha \theta}}{\left(1+\left(\theta_{w}-1\right) \theta\right)} \operatorname{Re} B r \\
& +\frac{\operatorname{Re} B r\left(M+D_{a} e^{-\alpha \theta}\right)}{\left(1+\left(\theta_{w}-1\right) \theta\right)} f^{\prime 2}+\left(\frac{\gamma_{2} \operatorname{Re} \delta^{2}}{(1+\delta \phi)} \phi^{\prime 2}+\frac{\gamma_{2} \operatorname{Re} \delta\left(\theta_{w}-1\right)}{\left(1+\left(\theta_{w}-1\right) \theta\right)} \phi^{\prime} \theta^{\prime}\right) .
\end{aligned}
$$


Where $N_{G}$ indicates summation of all the sources of entropy generation which are the respective terms on the right hand side of Eq. (2.14): Heat Transfer (HT), Fluid Friction (FF), Magnetic/Joule heating (MJH) and diffusive irreversibility/mass transfer (DIR). Meanwhile, the measure of contributions of each of these sources is defined by the Bejan number $B e$. This important quantity describes the ratio of the irreversibility owing to heat and mass transfer $(H T+D I R)$ to the entropy generation number $\left(N_{G}\right)$. Mathematically, it is defined as:

$$
B e=\frac{H T+D I R}{N_{G}}=\frac{H T+D I R}{H T+F F+M J H+D I R} .
$$

The emerging parameters in the dimensionless equations are respectively listed in (2.16) as temperature ratio, Prandtl number, magnetic field parameter, radiation parameter, Eckert number, Schmidt number, entropy generation number, Biot number, characteristic entropy generation, Brownian motion term, thermophoresis parameter, velocity ratio parameter, Weissenberg number, local Reynolds number, concentration relative term, Brikman number and Darcy number.

$$
\begin{aligned}
& \theta_{w}=\frac{T_{f}}{T_{\infty}}, \operatorname{Pr}=\frac{\mu_{\infty} c_{p}}{k_{\infty}}, M=\frac{\sigma B_{0}^{2}}{b \rho}, N r=\frac{16 \sigma^{\star} T_{\infty}^{3}}{3 k^{\star} k_{\infty}}, E c=\frac{U_{w}^{2}}{C_{p}\left(T_{w}-T_{\infty}\right)}, S c=\frac{\nu_{\infty}}{D_{B}}, N_{G}=\frac{S_{g e n}}{E_{g c}} \\
& \zeta=\frac{h_{f}}{k_{\infty}} \sqrt{\frac{\nu_{\infty}}{b}}, E_{g c}=\frac{k_{\infty}}{x^{2}}, N b=\frac{\left(\rho c_{p}\right)_{p} D_{B}\left(C_{w}-C_{\infty}\right)}{\left(\rho c_{p}\right)_{f} \nu}, N t=\frac{\left(\rho c_{p}\right)_{p} D_{T}\left(T_{w}-T_{\infty}\right)}{\left(\rho c_{p}\right)_{f} T_{\infty} \nu}, H=\frac{c}{b}, \\
& W e=\Gamma \sqrt{2 \frac{c^{3} x^{3 r-1}}{\nu_{\infty}}}, R e_{x}=\frac{U_{w} x}{\nu_{\infty}}, \delta=\frac{\left(C_{f}-C_{\infty}\right)}{C_{\infty}}, B r=\frac{\mu_{\infty} U_{w}^{2}}{k_{\infty} T_{\infty}}, D_{a}=\frac{\nu_{\infty}}{b K_{0}} .
\end{aligned}
$$

The quantities of engineering interest are respectively presented in (2.17)

$$
C_{f x}=\frac{2 \tau_{w}}{\rho_{\infty} U_{w}^{2}}, N u_{x}=\frac{x q_{w}}{k_{\infty}\left(T_{f}-T_{\infty}\right)}, S h_{x}=\frac{x q_{m}}{D_{B}\left(C-C_{\infty}\right)},
$$

with $C_{f x}, N u_{x}$ and $S h_{x}$ respectively corresponds to the coefficient of skin friction, local Nusselt and Sherwood numbers. Also

$$
\tau_{w}=\mu_{\infty}\left[\frac{\partial u}{\partial y}+\frac{\Gamma}{\sqrt{2}}\left(\frac{\partial u}{\partial y}\right)^{2}\right]_{y=0}, q_{w}=-\left.\left(k_{\infty}+\frac{16 T^{3} \sigma^{\star}}{3 k^{\star}}\right) \frac{\partial T}{\partial y}\right|_{y=0}, q_{m}=-\left.D_{B} \frac{\partial C}{\partial y}\right|_{y=0},
$$

where $\tau_{w}, q_{w}$ and $q_{m}$ denotes the surface shear stress, heat flux and mass flux respectively. The dimensionless skin friction coefficient is

$$
C_{f x}^{\star}=\frac{1}{2} R e^{\frac{1}{2}} C_{f x}=\left(f^{\prime \prime}(0)+\frac{W e}{2} f^{\prime \prime 2}(0)\right),
$$

while $N u_{x}$ and $S h_{x}$ respectively translate to

$$
N u_{x}^{\star}=R e_{x}^{-\frac{1}{2}} N u_{x}=-\left[1+N r\left(1+\left(\theta_{w}-1\right) \theta(0)\right)^{3}\right] \theta^{\prime}(0), S h_{x}^{\star}=R e_{x}^{-\frac{1}{2}} S h_{x}=-\phi^{\prime}(0) .
$$

\section{Method of Solution}

The boundary value problem (2.10-2.13) is numerically solved by shooting technique associated with Runge-Kutta-Fehlberg method. This becomes necessary due to the nonlinearity nature of the system of equations (2.10-2.12). The details of this method can be found in Mabood et al. [45] as well as Fatunmbi and Okoya [1]. A comparative analysis has been carried out with previous studies in the limiting situation with respect to $C_{f x}^{\star}$ for changes in $M$ as recorded in Table 1. The comparison has been done with the works of Mabood \& Das [46] as well as and Xu \& Lee [47] which validate our code. The default values for the plotting of the figures and tables are set as $W e=\varepsilon=$ 
$N r=E c=N_{b}=N_{t}=0.1, r=1.0, \zeta=0.2, \theta_{w}=1.3, S c=1.0, \operatorname{Pr}=2.0, \alpha=H=M=D_{a}=0.2$, except otherwise mentioned in the graphs.

Table 1: Computational values of $C_{f x}^{\star}$ as compared with [46] and [47] for variation in $M$

\begin{tabular}{cccc}
\hline $\mathrm{M}$ & {$[43]$} & {$[44]$} & Present Study \\
\hline 0 & 1.00001 & - & 1.00000 \\
1 & 1.41421 & 1.41421 & 1.41421 \\
5 & 2.44949 & 2.44941 & 2.44949 \\
10 & 3.31662 & 3.31660 & 3.31662 \\
50 & 7.14143 & 7.14140 & 7.14143 \\
100 & 10.04988 & 10.04980 & 10.04989 \\
500 & 22.38303 & 22.38301 & 22.38302 \\
1000 & 31.63858 & - & 31.63863 \\
\hline
\end{tabular}

\section{Results and Discussion}

The impacts of selected parameters on the skin friction coefficient $C_{f x}^{\star}$ have been displayed in Table 2. These parameters are: viscosity variation $\alpha$, velocity ratio $H$, Weissenberg number $W e$, magnetic field $M$ and Darcy number $D_{a}$. Clearly, there is a rise in $C_{f x}^{\star}$ as the magnitude of $W e, M$ and $D_{a}$ increases as observed in Table 2. A rise in the viscosity term creates a resistance in the flow field leading higher friction in the fluid particles. The viscous drag can be reduced by lowering the magnitude of these parameters so as to achieve the desired quality. On the contrary, a hike in the velocity ratio parameter $H$, there is a reduction in the skin friction coefficient $C_{f x}^{\star}$ as noticed in Table 2 .

Table 2: Computed values of $C_{f x}^{\star}$ for diverse values of $\alpha, H, W e, M$ and $D_{a}$

\begin{tabular}{cccccc}
\hline$\alpha$ & $H$ & $W e$ & $M$ & $D_{a}$ & $C_{f x}^{\star}$ \\
\hline 0.1 & 0.2 & 0.2 & 0.2 & 0.2 & 0.858082 \\
0.3 & & & & & 0.895227 \\
0.6 & & & & & 0.914454 \\
0.2 & 0.3 & & & & 0.778375 \\
& 0.6 & & & & 0.462723 \\
& 0.2 & 0.1 & & & 0.813508 \\
& & 0.2 & & & 0.876442 \\
& & 0.3 & & & 0.945947 \\
& & 0.2 & 0.0 & & 0.756424 \\
& & & 0.5 & & 1.037677 \\
& & & 1.0 & & 1.274356 \\
& & & 0.2 & 0.2 & 0.876442 \\
& & & & 0.4 & 0.978448 \\
& & & & 0.6 & 1.072739 \\
\hline
\end{tabular}

Table 3: Computational values of $N u_{x}^{\star}$ for varying $\alpha, \zeta, N r, \theta_{w}$ and $Q$ 


\begin{tabular}{cccccc}
\hline$\alpha$ & $\zeta$ & $N r$ & $\theta_{w}$ & $N u_{x}^{\star}$ & \\
\hline 0.1 & 0.2 & 0.2 & 1.3 & 0.2 & 0.147751 \\
0.3 & & & & & 0.147727 \\
0.6 & & & & & 0.147645 \\
0.2 & 0.1 & & & & 0.085974 \\
& 0.6 & & & & 0.279482 \\
& 1.0 & & & & 0.337940 \\
& 0.2 & 0.1 & & & 0.131978 \\
& & 0.2 & & & 0.147743 \\
& & 0.3 & & & 0.163368 \\
& & 0.2 & 1.2 & & 0.144484 \\
& & & 1.5 & & 0.155064 \\
& & & 1.8 & & 0.168305 \\
\hline
\end{tabular}

To further determine the effects of some parameters on the heat transfer rates across the surface of the sheet Table 3 has been constructed. From this table, it is noted an increase in the magnitude of thermal conductivity term $\zeta$, thermal radiation parameter $N r$ and temperature ratio parameter $\theta_{w}$ raises the transfer of heat at the surface of the sheet whereas a slight reduction is found in $N u_{x}^{\star}$ for a rise in the viscosity variation parameter.

\subsection{Parameters effect on velocity profiles}

The graph of velocity versus $\eta$ for variations in $M$ and $H$ are displayed in Fig. 2a. As noticed in this figure, a boost in $M$ creates a drag in the fluid motion due to the action of the Lorentz force. An application of the transverse magnetic field to the electrically conducting Williamson fluid generates Lorentz force which is responsible for the resistance in the fluid motion and at such, a reduction in the velocity as depicted in this figure. However, this trend is reversed for higher $H$, a rise in the velocity ratio $H$ enhances the motion of the Williamson nanofluid. In the absence of $H$, the velocity is raised, in this view, the flow of the fluid can be controlled effectively by adjusting the magnitude of the velocity ratio term $H$. Fig, $2 \mathrm{~b}$ depicts the impact of Darcy number $D_{a}$ and that of the nonlinear stretching term $r$ on the velocity field. Clearly, there is a decelerated flow for growth in both $D_{a}$ and $r$. With a rise in $D_{a}$, a resistance to flow is encountered. At the same time, the nonlinearity of the stretching sheet impedes the flow by raising the friction between the fluid and the sheet. Likewise, the velocity reduces for a rise in the viscosity term $\alpha$ and Weissenberg number $W e$ as displayed in Fig. 2c. A rise in $\alpha$ strengthens the viscous force which in turn lowers the fluid motion. Similarly, a rise in the relaxation time occurs with higher $W e$ which consequently creates a drag in the flow field.
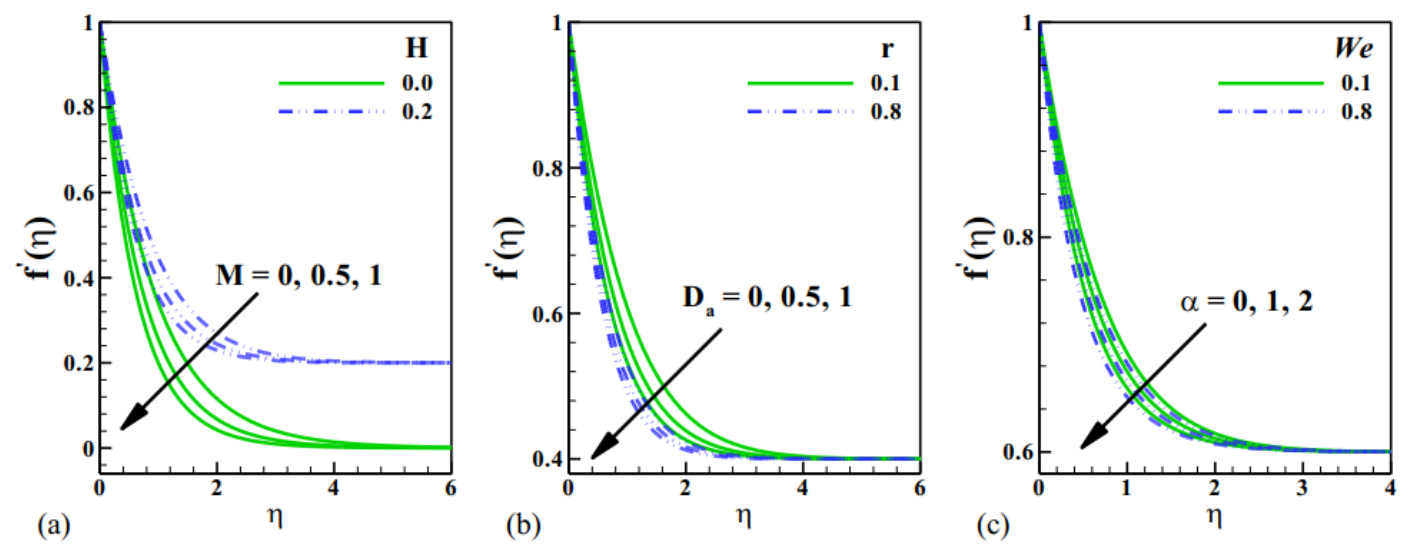
Fig. 2 Velocity profile for changes in (a) $M$ and $H$ (b) $D a$ and $r$ (c) $\alpha$ and $W e$

\subsection{Parameters effect on temperature profiles}

The effects of some parameters namely: magnetic field $M$, Darcy number $D_{a}$ and thermal radiation $N r$ with respect to temperature distribution are portrayed in Figs 3a-c in the presence of $H, r$ and $\alpha$ respectively. In Fig. 3a, the temperature is raised with higher $M$ due to extra heating created by Lorentz force. On the other hand, a rise in $H$ lowers the surface temperature due to a fall in the friction between the fluid and the surface. Higher values of $H$ improves heat transfer such that the nonlinearly stretched sheet takes less time to cool.
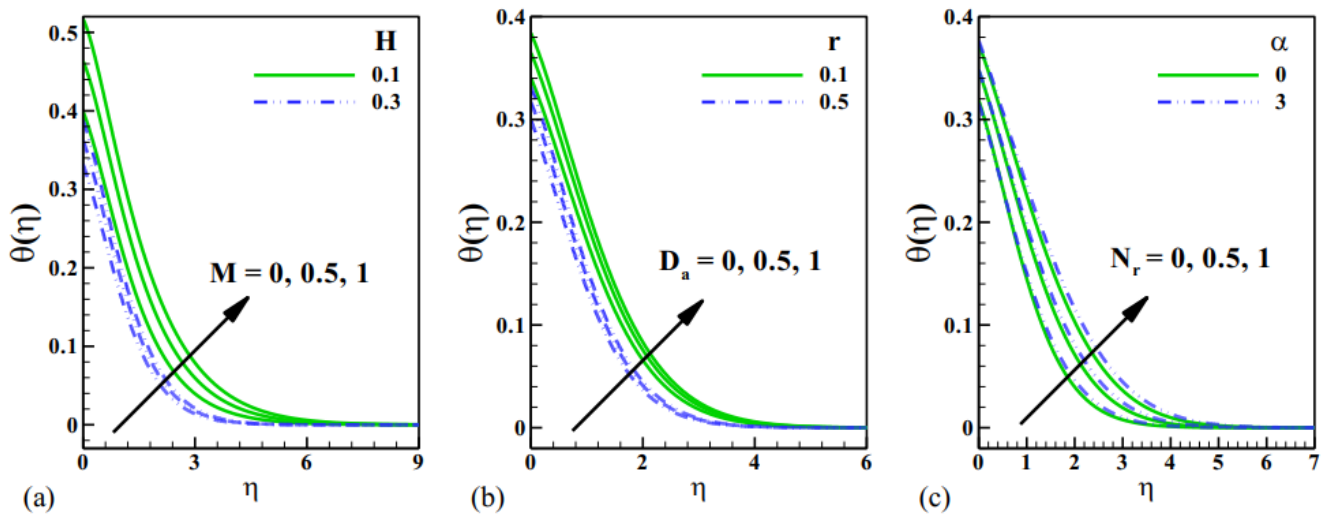

Fig. 3 Temperature distribution for the changes in (a) $M$ and $H$ (b) $D_{a}$ and $r$ (c) $N r$ and $\alpha$

The thermal boundary structure shrinks with arise in $\operatorname{Pr}$ as shown in Fig 4a. The ratio of momentum diffusivity to that of thermal diffusivity is described by $P r$. Thus, with a rise in $P r$, the thermal boundary layer declines and in consequence, the average temperature reduces. However, a rise in the thermal conductivity term $\varepsilon$ strengthens the temperature distribution as found in Fig. 4a. Also a hike in $\theta_{w}$ favours the operating temperature $\left(T_{f}-T_{\infty}\right)$ which in turn propels a rise in the fluid temperature as seen in Fig. 4b. Likewise, an increase in the Eckert number Ec causes thickness of the thermal boundary layer to swell up due to the friction between the fluid particles and consequently, the temperature distribution is increased as noticed in Fig. 4b. 


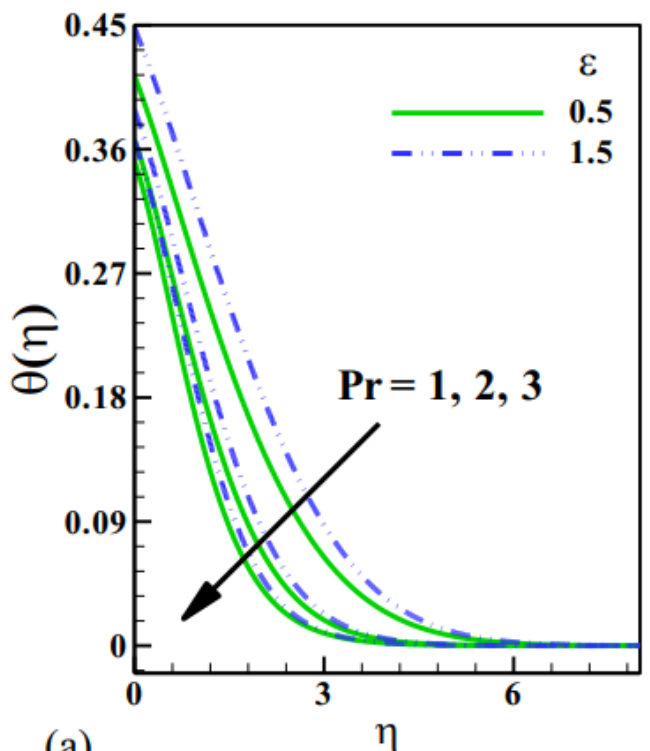

(a)

Fig. 4 Temperature profiles for the changes in (a) $\operatorname{Pr}$ and $\varepsilon$ (b) $\theta_{w}$ and $E c$

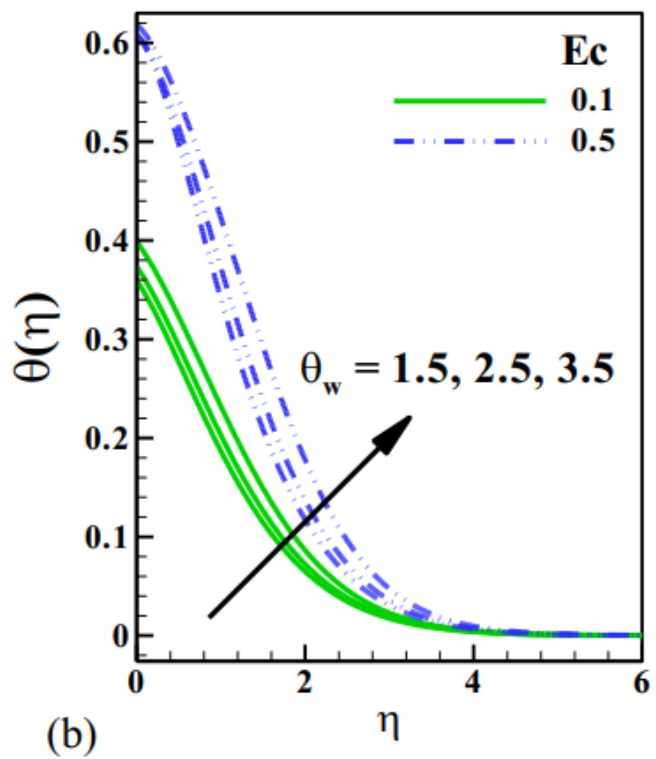

\subsection{Parameters reactions on nanoparticles concentration field}

Figures 5(a-c) describe the response of the nanoparticles concentration field to variations in the magnetic field term $M$, thermophoresis term $N t$ and Schmidt number $S c$ in the presence of velocity ratio term $H$, Brownian motion $N b$ and nonlinear stretching parameter $r$ respectively. In Fig. 5a, the concentration profile is raised with growth in $M$ whereas a declining nature is observed with a rise in $H$. An enlargement in the thermophoresis term $N_{t}$ compels an expansion in the concentration boundary layer and at such energizes the concentration profiles as demonstrated in Fig. $5 \mathrm{~b}$.

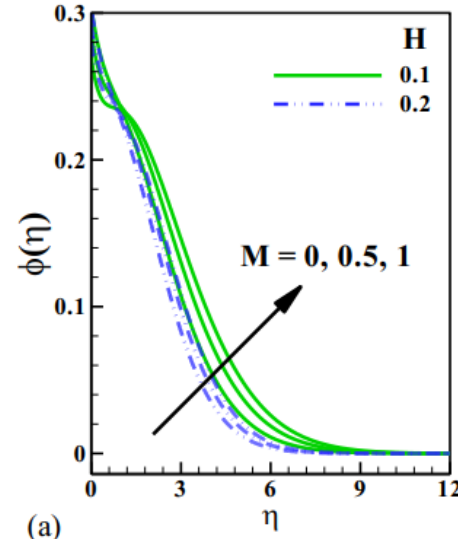

(a)

Fig. 5 Concentration profiles for variation in (a) $M$ and $H$

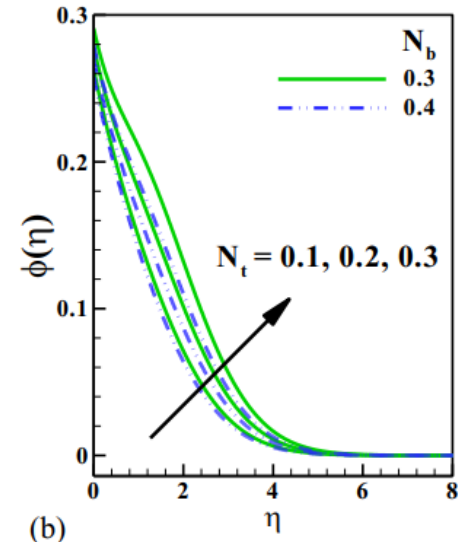

(b)

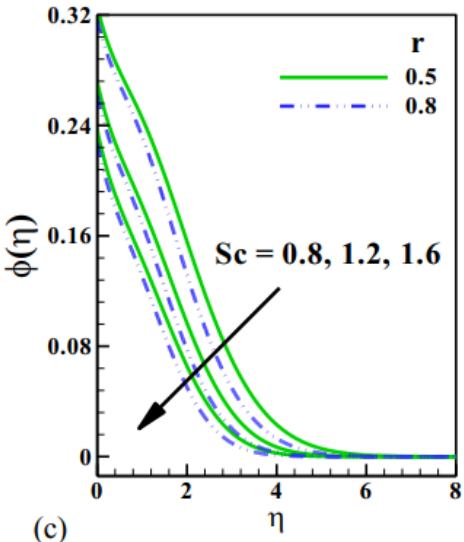

(c) 


\subsection{Parameters effects on entropy generation and Bejan number}

The reactions of the entropy generation $N_{G}$ as dependent on some parameters are revealed in Fig $6(\mathrm{a}-\mathrm{c})$. Evidently, a rise in $\delta$ and $M$ promotes an increase in $N_{G}$. The diffusion parameter due to nanoparticles concentration $\gamma_{2}$ likewise strengthens $N_{G}$ as shown in Fig. 6a. Meanwhile, an increase in $r$ reduces $N_{G}$ while $H$ enhances it as noted in Fig. 6b. More so, the entropy generation $N_{G}$ advances with increasing values of $\gamma_{2}$ and $S c$ but a rise in the Biot number $\zeta$ lowers it as displayed in Fig. 6c.

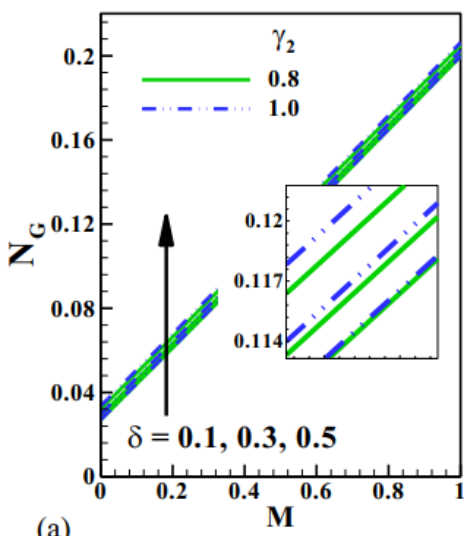

(a)

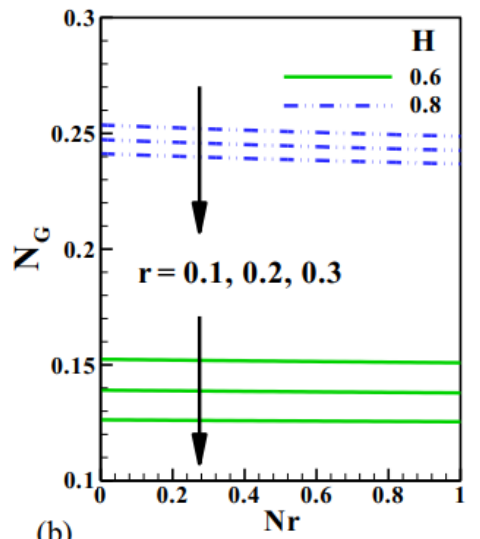

(b)

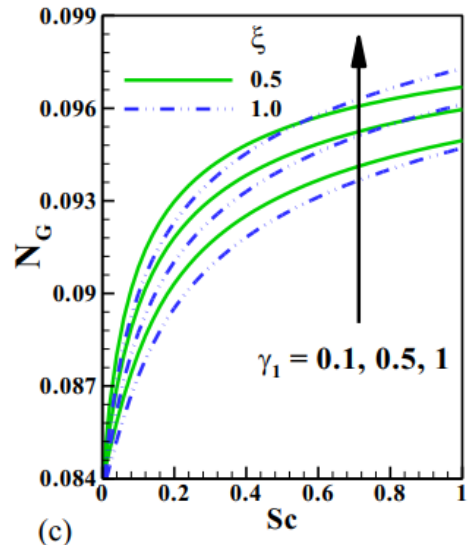

(c)

(c) $\gamma_{2}, \zeta \& S c$

The reaction of Bejan number $B e$ for variations in $\delta$ and diffusion term $\gamma_{2}$ is depicted in Fig. 7a. The trend informs that entropy generation resulting from heat and mass transfer dominates over that of the magnetic and fluid friction irreversibilities due to a rise in $B e$ as $\delta$ and $\gamma_{2}$ increases. However, for any value of $\delta$, a rise in $M$ reduces $B e$ and such implies that the frictional and magnetic irreversibilities is stronger than heat and mass transfer irreversibilities. In Fig. 7b, Be advances with a rise $r$ and $N r$. This trend implies the dominance of heat and mass transfer irreversibilities while the opposite occurs with a hike in $H$. In Fig. $7 \mathrm{c}$, it is noticed that the irreversibilities due to heat and transfer dominate in the presence of $\gamma_{2}, \zeta$ and $S c$ due to a rise in $B e$.
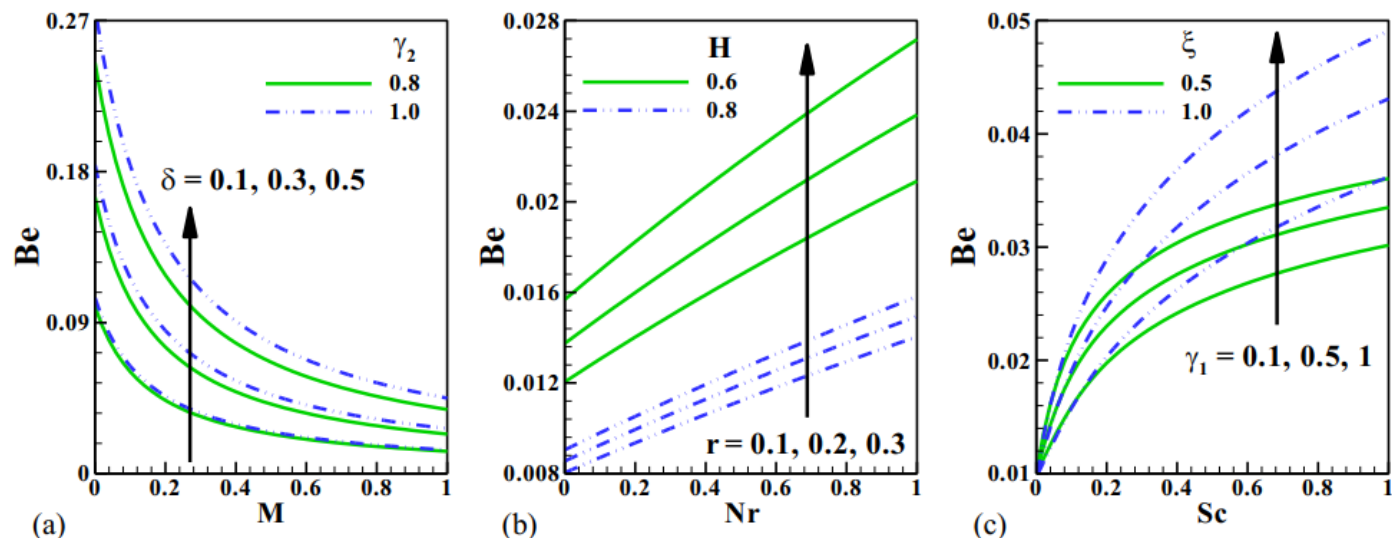

Fig. 7 Combined reactions of (a) $\gamma_{2}, \delta \& M$ (b) $r, H \& N r$ and (c) $\gamma_{2}, \zeta \& S c$ on $B e$

\section{Conclusion}

The present analysis investigates stagnation point flow of magneto-Williamson nanofluid flow over a convectively heated nonlinearly stretched sheet in a porous medium with entropy generation 
analysis. The flow is characterized by temperature-dependent thermophysical properties coupled with the impact of nonlinear thermal radiation and viscous dissipation. The equations that describe the physical model are further simplified by means of relevant similarity transformation variables and then integrated by the Runge-Kutta Fehlberg algorithm. The solutions are presented in the form of graphs and tables for better explanation while the results are in good term with existing studies available in literature for limiting situations. The investigation reveals that:

- A raise in the magnitude of the magnetic field parameter, viscosity parameters as well as Weissenberg number enhances skin friction coefficient whereas the opposite is the case with a rise in the velocity ratio term.

- The heat transfer improves with a rise in the thermal conductivity parameter, radiation, temperature ratio and velocity ratio parameter while it diminishes with higher magnetic field parameter.

- Entropy generation advances with a rise in the temperature relative parameter, the magnetic term and velocity ratio term and diffusion term due to nanoparticle as well as velocity ratio term. Thus minimization of entropy can be achieved by lowering the magnitude of these parameters.

- The entropy generation resulting from heat and mass transfer has a dominance effect over that of magnetic and fluid friction as thermal radiation term, diffusion term and nonlinear stretching term $r$ are raised whereas the trend is reversed with growth in the magnetic field and velocity ratio parameters.

\section{Acknowledgements}

The authors are grateful to the authors referred in this work.

\section{References}

[1] Fatunmbi, E. O., Okoya, S. S. Heat transfer in boundary layer magneto-micropolar Fluids with temperature-dependent material properties over a stretching sheet, Advances in Materials Science and Engineering, 2020, Article ID 5734979, 11 pages, https://doi.org/10.1155/2020/5734979

[2] Williamson, R. V. The flow of pseudoplastic materials, Industrial and Engineering Chemistry, 21(11), 1108-1111 (1929).

[3] Nadeem, S., Hussain, S. T. and Lee, C. Flow of a Williamson fluid over a stretching sheet, Brazilian Journal of Chemical Engineering, 30(3), 615-625 (2013) .

[4] Shah, Z., Bonyah, E., Islam, S., Khan, W., Ishaq, M. Radiative MHD thin film flow of Williamson fluid over an unsteady permeable stretching, Heliyon, 4 (2018) e00825. doi: 10.1016/j.heliyon.2018.e00825

[5] Qayyum, S., Khan, M. I., Hayat, T., Alsaedi, A.,. Tamoor, M. Entropy generation in dissipative flow of Williamson fluid between two rotating disks, International Journal of Heat and Mass Transfer. 127, 933-942 (2018).

[6] Aldabesh, A., Khan, S. U.Habib, D., et al.Unsteady transient slip flow of Williamson nanofluid containing gyrotactic microorganism and activation energy, Alexandria Engineering Journal 59, 4315-4328 (2020).

[7] Crane, L. J. Flow past a stretching plate. Communicatios Breves, 21, 645-647 (1970). 
[8] Qasim, M., Khan, I. and Shafie, S. Heat Transfer in a Micropolar Fluid over a Stretching Sheet with Newtonian Heating, PLoS ONE 8(4) 1-6, (2013).

[9] Kumar, L. Finite element analysis of combined heat and mass transfer in hydromagnetic micropolar flow along a stretching sheet. Comput Mater Sci, 46, 841-848 (2009).

[10] Akinbobola, T. E. and Okoya, S. S. The flow of second grade fluid over a stretching sheet with variable thermal conductivity and viscosity in the presence of heat source/sink, Journal of the Nigerian Mathematical Society, 34, 331-342 (2015).

[11] Fatunmbi, E. O., Fenuga, O. J. (2018). MHD micropolar fluid fow over a permeable stretching sheet in the presence of variable viscosity and thermal conductivity with Soret and Dufour effects. International Journal of Mathematical Analysis and Optimization: Theory and Application, 2017, 211- 232 (2017).

[12] Gupta, P. S., Gupta, A. S. Heat and mass transfer on a stretching sheet with suction or blowing, Canad. J. Chem. Eng. 55, 744-746 (1977). Vajravelu, K. Viscous flow over a nonlinearly stretching sheet, Appl. Math. Comput. 124, 281-288 (2001).

[13] Cortell, R., Viscous flow and heat transfer over a nonlinearly stretching sheet, Appl. Math. Comput. 184, 864-873 (2007)

[14] Hayat, T, Abbas, Z., Javed, T. Mixed convection flow of a micropolar fluid over a non-linearly stretching sheet, Phys. Lett. 372, 637-647 (2008) .

[15] Waqas, M., Farooq,M.,, Khan, M. J., Alsaedi, A., Hayat, T., Yasmeen, T. Magnetohydrodynamic (MHD) mixed convection flow of micropolar liquid due to nonlinear stretched sheet with convective condition, Int. J. Heat Mass Tran. 102, 766-772 (2016).

[16] Fatunmbi, E.O., Okoya, S. S. and Makinde, O. D. Convective heat transfer analysis of hydromagnetic micropolar fluid flow past an inclined nonlinear stretching sheet with variable thermo-physical properties. Diffusion Foundations, 26, 63-77 (2020).

[17] Noor, N. F. M., Haq, R. U., Nadeem, S., Hashim, I. Mixed convection stagnation flow of a micropolar nanofluid along a vertically stretching surface with slip effects, Meccanica, 50, 2007-2022 (2015).

[18] Aly, E. H., Pop, I. MHD flow and heat transfer near stagnation point over a stretching/shrinking surface with partial slip and viscous dissipation: Hybrid nanofluid versus nanofluid, Powder Technology, 367, 192-205 (2020).

[19] Anuar, N. S., Bachok, N., Pop, I. Cu-Al2O3/Water hybrid nanofluid stagnation point flow past MHD stretching/shrinking sheet in presence of homogeneous-heterogeneous and convective boundary conditions, Mathematics, 8, 1-14, (2020).

[20] Fatunmbi, E. O., Okoya, S. S. Quadratic mixed convection stagnation-point flow in hydromagnetic Casson nanofluid over a nonlinear stretching sheet with variable thermal conductivity, Defect and Diffusion Forum, 409, 95-109 (2021).

[21] Gbadeyan, J. A., Titiloye, E. O. and Adeosun, A. T. Effect of variable thermal conductivity and viscosity on Casson nanofluid flow with convective heating and velocity slip. Heliyon 6 , $1-10(2020)$.

[22] Mabood, F., Ibrahim, S. M. and Khan, W. A. Effect of melting and heat generation/absorption on Sisko nanofluid over a stretching surface with nonlinear radiation. Physica Scripta, 94(6) (2019). 
[23] Mukhopadhyay, S., Bhattacharyya, K. and Layek, G. C. Forced convective flow and heat transfer over a porous plate in a Darcy-Forchheimer porous medium in presence of radiation, Meccanica, 47, 153-161 (2012).

[24] Jafar, A. B., Shafie, S. Ullah, I. MHD radiative nanofluid flow induced by a nonlinear stretching sheet in a porous medium, Heliyon 6 (2020) e04201

[25] Fatunmbi, E. O. and Adeniyan, A. MHD stagnation point-flow of micropolar fluids past a permeable stretching plate in porous media with thermal radiation, chemical reaction and viscous Dissipation, Journal of Advances in Mathematics and Computer Science,26(1), 1-19 (2018).

[26] Fatunmbi, E. O., Ogunseye, H. A. and Sibanda, P. Magnetohydrodynamic micropolar fluid flow in a porous medium with multiple slip conditions, International Communications in Heat and Mass Transfer, 115, 1-10 (2020).

[27] Bejan, A. Second law analysis in heat transfer and thermal design, Adv. Heat Transfer, 15, $1-58(1982)$.

[28] Bejan, A. Entropy generation minimization, (second ed.) CRC, New York (1996).

[29] Salawu, S. O. and Ogunseye, H. A. Entropy generation of a radiative hydromagnetic PowellEyring chemical reaction nanofluid with variable conductivity and electric field loading, Results in Engineering 5, 1-8 (2020).

[30] Sithole, H., Mondal, H. and Sibanda, P. Entropy generation in a second grade magnetohydrodynamic nanofluid flow over a convectively heated stretching sheet with nonlinear thermal radiation and viscous dissipation, Result in Physics, 9, 1077-1085 (2018).

[31] Mondal, H., Mishra, S., Kundu, P. K. and Sibanda, P. Entropy generation of variable viscosity and thermal radiation on magneto nanofluid flow with dusty fluid, J. Appl. Comput. Mech., 6(1), 171-182 (2020).

[32] Shit, G. C. and Mandal, S. Entropy analysis o unsteady MHD flow of Casson nanofluid over a stretching vertical plate with thermal radiation effects, Int. J. Appl. Comput. Math, 6(2), 1-22 (2020).

[33] Fatunmbi, E. O. and Salawu, S. O. Thermodynamic second law analysis of magneto-micropolar fluid flow past nonlinear porous media with non-uniform heat source, Propulsion and Power Research, 9(3), 1-8 (2020).

[34] Alsaadi, F. E., Hayat, T., Khan, M. I. and Alsaadi, F. E. Heat transport and entropy optimization in flow of magneto-Williamson nanomaterial with Arrhenius activation energy, Computer Methods and Programs in Biomedicine, 183, 1-8 (2020).

[35] Fatunmbi, E. O. and Salawu, S. O. Analysis of entropy generation in hydromagnetic micropolar fluid flow over an inclined nonlinear permeable stretching sheet with variable viscosity, J. Appl. Comput. Mech., 6(SI), 1301-1313 (2020). DOI: 10.22055/JACM.2019.30990.1807

[36] Fatunmbi, E. O., Adeniyan, A. Nonlinear thermal radiation and entropy generation on steady flow of magneto-micropolar fluid passing a stretchable sheet with variable properties, Results in Engineering, 6, 1-10, (2020).

[37] Rashidi, M. M., Ali, M., Rostami, B., Rostami, P. and Xie, G. Heat and Mass Transfer for MHD Viscoelastic Fluid Flow over a Vertical Stretching Sheet with Considering Soret and Dufour Effects, Mathematical Problems in Engineering, 2015, 1-12 (2015). 
[38] Mabood, F., Khan, W. A., Ismail, A.I.M. MHD boundary layer flow and heat transfer of nanofluids over a nonlinear stretching sheet: A numerical study, Journal of Magnetism and Magnetic Materials 374, 569-576 (2015).

[39] Bhargava, R., Chandra, H. Numerical simulation of MHD boundary layer flow and heat transfer over a nonlinear stretching sheet in the porous medium with viscous dissipation using hybrid approach, arXiv preprint arXiv, 711.03579, 1-19 (2017).

[40] Isede, H. A. and Adeniyan, A. Dissipative nanofluid slip-flow and heat transfer in a permeable stretching vertical channel with internal heat generation. International Journal of Mathematical Analysis and Optimization Theory and Applications, 2020(1) 669-688 (2020)..

[41] Megahed, A. M. Williamson fluid flow due to a nonlinearly stretching sheet with viscous dissipation and thermal radiation, Journal of the Egyptian Mathematical Society, 27:12 (2019).

[42] Afridi, M. I., Qasim, M., Khan, I. Entropy generation in MHD mixed convection stagnationpoint flow in the presence of joule and frictional heating, Case Studies in Thermal Engineering, 12:292-300 (2018).

[43] Jamalabadi, M. Y. A. Entropy generation in boundary layer flow of a micropolar fluid over a stretching sheet embedded in a highly absorbing medium, Frontier in Heat and Mass Transfer, 6(7), 1-13 (2015).

[44] Baag, S., Mishra, S.R., Dash, G.C. and Acharya, M.R. Entropy generation analysis for viscoelastic MHD flow over a stretching sheet embedded in a porous medium, Ain Shams Engineering Journal, doi.org/10.1016/j.asej.2015.10.017

[45] Mabood, F., Ibrahim, S. M., Rashidi, M. M., Shadloo, M. S. and Lorenzini, G. Non-uniform heat source/sink and Soret effects on MHD non-Darcian convective flow past a stretching sheet in a micropolar fluid with radiation. Int. J. Heat Mass Transfer 93, 674-682 (2016).

[46] Mabood, F. and Das, K. Melting heat transfer on hydromagnetic flow of a nanofluid over a stretching sheet with radiation and second-order slip, Eur. Phys. J. Plus, 131, 3 (2016).

[47] Xu, L., Lee, E.W.M. Variational iteration method for the magnetohydrodynamic flow over a nonlinear stretching sheet. Abst. Appl. Anal. 5 pages (2013). 